\title{
TITLE:
}

\section{Characterization of liquid cluster ion source by the time-of-flight method}

\section{AUTHOR(S):}

Takaoka, GH; Noguchi, H; Nakayama, K; Kawashita, $\mathrm{M}$

\section{CITATION:}

Takaoka, GH ...[et al]. Characterization of liquid cluster ion source by the time-of-flight method. REVIEW OF SCIENTIFIC INSTRUMENTS 2006, 77(3): 03B509.

\section{ISSUE DATE:}

2006-03

URL:

http://hdl.handle.net/2433/50111

\section{RIGHT:}

Copyright 2006 American Institute of Physics. This article may be downloaded for personal use only. Any other use requires prior permission of the author and the American Institute of Physics. 


\title{
Characterization of liquid cluster ion source by the time-of-flight method
}

\author{
Gikan H. Takaoka, Hidetaka Noguchi, Kazuya Nakayama, and Masakazu Kawashita \\ Ion Beam Engineering Experimental Laboratory, Kyoto University, Nishikyo, Kyoto 615-8510, Japan
}

(Presented on 15 September 2005; published online 22 March 2006)

\begin{abstract}
A liquid cluster ion source, which offers many possibilities for new and advanced applications in the surface processing of materials, was developed, and the cluster size distribution was measured by using time-of-flight method. When the vapor pressure of liquid materials such as ethanol and water was larger than $1 \mathrm{~atm}$, the liquid clusters could be produced by an adiabatic expansion phenomenon. The peak size of the cluster ions increased with the increase of the vapor pressures, and decreased with the increase of the electron voltage and the electron current for ionization. In another case of producing liquid cluster ions at lower vapor pressures, He gas was used to mix up with vapors of liquid materials. Even if a vapor pressure was less than $0.1 \mathrm{~atm}$, the liquid cluster ions with smaller size could be produced at the $\mathrm{He}$ gas pressures larger than $0.5 \mathrm{~atm}$. The vapors of the liquid materials were cooled down by the collision with the He gas, which resulted in the effective formation of the liquid clusters even at lower vapor pressures. (C) 2006 American Institute of Physics. [DOI: 10.1063/1.2172345]
\end{abstract}

\section{INTRODUCTION}

A cluster is an aggregate of a few tens to several thousands of atoms, and the physical and chemical properties of the cluster are different from those of bulk state. ${ }^{1-4}$ As well as the physical and chemical properties of the cluster itself, the interaction of energetic cluster ions with solid surface atoms is very attractive. High-energy-density deposition and collective motions of the cluster ions on the solid surface play important roles in the surface modification by cluster ion beams. ${ }^{5-7}$ In addition, a cluster ion has an extremely low charge-to-mass ratio. Since the number of charges in the cluster ion is much smaller than the number of atoms, a cluster ion can transport thousands of times larger numbers of atoms at the same ion current compared with a monomer ion. For example, if the cluster ion current with cluster size of 1000 is $1 \mu \mathrm{A}$, it can transport the same number of atoms as a $1 \mathrm{~mA}$ monomer ion beam. Furthermore, since the kinetic energy of the atom in the cluster is equal to the total energy divided by the cluster size, low-energy ion beams can be realized using cluster ion beams at relatively high acceleration voltages.

The cluster ion beam process has attracted much interest as an advanced ion beam technology, which is one of the basic technologies for surface treatment. However, the engineering applications of the widely processing technology of surface treatment using cluster ion beams with liquid source materials have received less attention so far. Interest for liquid material ions have shown in mass spectroscopic studies. $^{8-11}$ Liquid materials including organic materials have several features, one of which is the presence of various kinds of structural and chemical properties. This feature is useful for the chemical modification of solid surfaces, because the chemical reaction on the surfaces is different depending on the liquid materials used. Another feature is that the inherent fluid property of liquid materials as well as gas materials is effective for their continuous supply to the ion source, which is very useful for the engineering applications of ion beam processes. From the point of the engineering applications of liquid materials with these features, it should be noted that large current ion beams are of much importance.

We produced liquid cluster ions such as ethanol cluster ions, ${ }^{12,13}$ and measured the cluster size distribution by using the retarding potential method. The mass resolution of this method is defined principally by the uniformity of the potential at the ionizing point and the retarding electrode. The energy resolution of a typical retarding electrode is about $10 \mathrm{eV}$, which corresponds to a size of a few hundreds of atoms per cluster. Therefore, the maximum mass resolution is less than 10 and not sufficient, but this method is a simple and easy way to measure the approximate cluster size distribution. The physical and chemical properties of the liquid clusters depend on the cluster size, and it is important to measure the cluster size distributions more accurately. In addition, the liquid clusters with cluster size less than a few hundreds of molecules per cluster are very attractive in order to investigate chemical reactions between cluster and surface atoms. In this article, the production of liquid clusters such as ethanol and water clusters is described, and the cluster size distribution is investigated by using the time-of-flight (TOF) method. Furthermore, the cluster formation by mixing the vapors of the liquid materials with $\mathrm{He}$ gas is discussed based on the TOF measurement.

\section{EXPERIMENTAL APPARATUS}

Figure 1 shows the schematic of the liquid cluster ion source combined with a TOF instrument. Liquid materials such as ethanol and water are introduced into a cluster source, and heated up to $150{ }^{\circ} \mathrm{C}$ by a wire heater attached around the source. The vapors of the liquid materials are injected through a nozzle into a high-vacuum region. The nozzle is made of glass, and it is a converging-diverging supersonic nozzle with a throat diameter of $0.1 \mathrm{~mm}$. Vapor pressure increases with the increase of the source temperature. For example, the vapor pressures of ethanol at the source temperatures of 78 and $109^{\circ} \mathrm{C}$ were 1 and $3 \mathrm{~atm}$, 


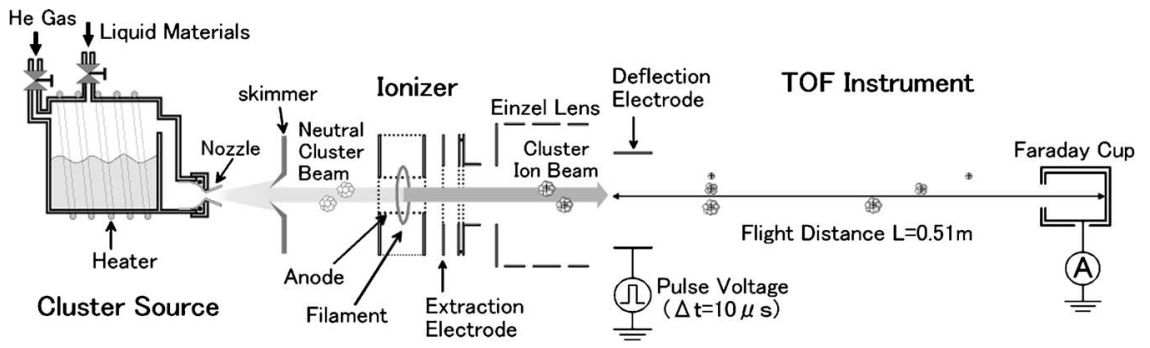

FIG. 1. Schematic of the liquid cluster ion source combined with the TOF instrument. respectively. When the vapor pressure is larger than 1 atm, the vaporized liquid clusters can be produced by an adiabatic expansion phenomenon. As shown in the figure, the clusters produced pass through a skimmer and enter an ionizer. In the ionizer, electrons are emitted from a hot filament of a tungsten wire and accelerated by a voltage applied between the filament and the cylindrical anode. The neutral clusters entering the anode are ionized by an electron bombardment method. The electron voltage for ionization $\left(V_{e}\right)$ was adjusted between 100 and $500 \mathrm{~V}$, and the electron current for ionization $\left(I_{e}\right)$ was adjusted between 50 and $500 \mathrm{~mA}$. The cluster ions are accelerated by applying an acceleration voltage. The acceleration voltage $\left(V_{a}\right)$ was adjusted between 0 and $6 \mathrm{kV}$. The ion current obtained was a few microamperes for ethanol and water cluster ions, respectively.

The accelerated cluster ions enter a TOF instrument, in which cluster size is measured. The accelerated ions are deflected by a pulsed negative voltage. When the pulsed voltage is applied, the ions drift toward a Faraday cup. The ions are detected by the Faraday cup. They are monitored by a digital oscilloscope, and the drift time is measured. The pulse voltage was $2 \mathrm{kV}$. The duration time and the repetition rate of the pulse voltage were $10 \mu \mathrm{s}$ and $100 \mathrm{~Hz}$, respectively. The drift distance was $0.51 \mathrm{~m}$. The background pressure around the Faraday cup was $2 \times 10^{-7}$ Torr, which was attained using a turbo-molecular pump. The cluster size is calculated based on the drift time ranging from microseconds to milliseconds, which is different depending on the mass. Taking account of the influence of the ionization position on the drift time, ${ }^{14}$ the maximum mass resolution is estimated to be approximately 500, which is much higher than that of the retarding method. Furthermore, it was assumed that the cluster ion is a singly charged ion in the calculation of the cluster size.

\section{RESULTS AND DISCUSSION}

The size distribution of ethanol and water cluster ions was investigated by changing the source temperature, and the cluster size was distributed between a few hundreds and a few tens of thousands. According to the thermodynamics and the gas dynamics, the cluster beam intensity $(I)$ produced depends on the source pressure $P_{0}$, nozzle diameter $D$, and temperature of the source materials $T_{0}$. It is described as follows: ${ }^{15,16}$

$$
I \propto P_{0} D\left(\frac{T_{b}}{T_{0}}\right)^{\gamma / \gamma-1},
$$

where $\gamma$ and $T_{b}$ are the specific heat ratio and the boiling temperature of the source materials, respectively. Further- more, the maximum of the mass intensity distribution, which is defined as the peak size of cluster ions, increases with the increase of the beam intensity.

Figure 2 shows the relationship between the parameter $(\psi)$ and the peak size for ethanol and water cluster ions, where $\psi$ is defined as $P_{0} D\left(T_{b} / T_{0}\right)^{\gamma / \gamma-1}$. The nozzle diameter $D$ was kept at $0.1 \mathrm{~mm}$. The variation of peak size measured as an error bar was within 100 molecules per cluster. As shown in the figure, the peak size is about 250-1000 molecules per cluster for the ethanol cluster ions and about 1000-3000 molecules per cluster for the water cluster ions, respectively. The peak size of cluster ions increases with the increase of the vapor pressure. Furthermore, with regards to the source temperature, the beam intensity is proportional to $\left(T_{b} / T_{0}\right)^{\gamma /(\gamma-1)}$. The values of $T_{b}$ and $\gamma$ are $78{ }^{\circ} \mathrm{C}$ and 1.13 for ethanol, and $100{ }^{\circ} \mathrm{C}$ and 1.33 for water, respectively. Therefore, the value of $\left(T_{b} / T_{0}\right)^{\gamma /(\gamma-1)}$ at a constant pressure for water is larger than that for ethanol. This indicates that the peak size of water cluster ions is larger than that of ethanol cluster ions.

The size distribution of cluster ions depends on the electron voltage and the electron current for ionization. ${ }^{17,18}$ The peak size of ethanol and water cluster ions decreased with the increase of the electron voltage and the electron current for ionization. When clusters are ionized by electron bombardment, multiply charged cluster ions as well as singly charged cluster ions are produced. ${ }^{19,20}$ Furthermore, the ionization efficiency increases with the increase of the cluster size, and the cluster ions with larger size has higher possibility of producing multiply charged cluster ions. On the other hand, the fragmentation of the multiply charged cluster ions also occurs due to the Coulomb repulsion force, because the molecules in the cluster are weakly bound by van der Waals forces. $^{21,22}$ While the multiply charged cluster ions such as doubly charged cluster ions increase with the increase of the electron voltage for ionization, the fragmentation of the dou-

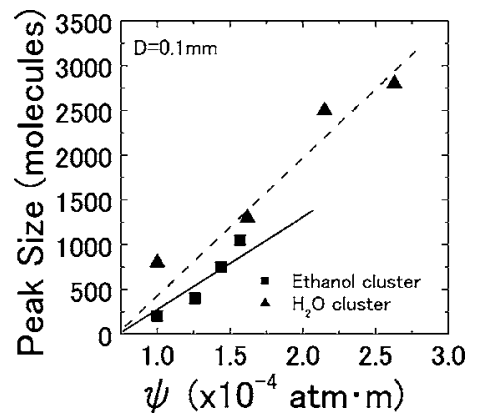

FIG. 2. Relationship between the parameter $(\psi)$ and the peak size for ethanol and water cluster ions. $\psi$ was defined as $P_{0} D\left(T_{b} / T_{0}\right)^{\gamma / \gamma-1}$. 

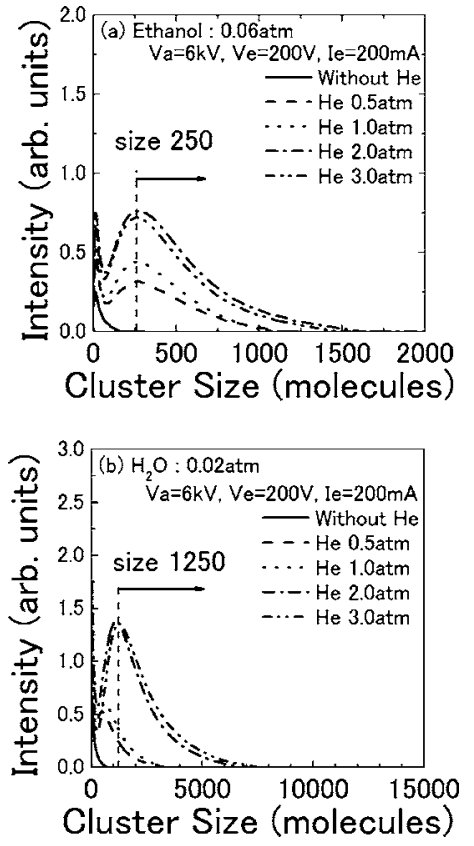

FIG. 3. Cluster size distribution for (a) ethanol and (b) water as a parameter of $\mathrm{He}$ gas pressure.

bly charged cluster ions is also enhanced. In addition, the production probability of doubly charged cluster ions might be larger than that of singly charged cluster ions at higher electron voltages for ionization, and the increase of electron current for ionization by keeping the electron voltage at a higher voltage such as a few hundreds of volts could enhance the production of doubly charged cluster ions. As a result, the fragmentation of the doubly charged cluster ions is also enhanced with increasing electron current for ionization. Therefore, the peak size decreased with the increase of the electron voltage and the electron current for ionization.

In another case of producing the liquid clusters, He gas was used to mix up with vapors of the liquid materials. Figure 3 shows a cluster size distribution for (a) ethanol and (b) water as a parameter of He gas pressure. The temperature of ethanol and water is room temperature, and the vapor pressure is $0.06 \mathrm{~atm}$ for ethanol and $0.02 \mathrm{~atm}$ for water, respectively. The electron voltage for ionization $\left(V_{e}\right)$ was $200 \mathrm{~V}$, and the electron current for ionization $\left(I_{e}\right)$ was $200 \mathrm{~mA}$. The acceleration voltage $\left(V_{a}\right)$ was $6 \mathrm{kV}$. As shown in Fig. 3, the intensity of ethanol and water clusters increases with the increase of the He gas pressure, even if the vapor pressure is low. The peak size of cluster ions is 250 molecules per cluster for the ethanol cluster ions and 1250 molecules per cluster for the water cluster ions, respectively. The total current of ethanol and water cluster ions were a few hundreds of nanoamperes at a $\mathrm{He}$ pressure of 2 and $3 \mathrm{~atm}$. When the ethanol and water vapors are injected through the nozzle into the high-vacuum region together with the He gas, they are cooled down by the collision with the He gas, which results in the effective formation of ethanol and water clusters even at the lower vapor pressure.

The vapors of the liquid materials such as ethanol and water were injected through a nozzle into a high-vacuum region. When the vapor pressure was larger than $1 \mathrm{~atm}$, the vaporized liquid clusters were produced by an adiabatic expansion phenomenon. The neutral clusters were ionized by an electron bombardment method, and the cluster size distribution was measured by the TOF method. The intensity of the cluster ion beams increased with the increase of the vapor pressures. The peak size was between 250 and 1000 molecules per cluster for the ethanol cluster ions and between 1000 and 3000 molecules per cluster for the water cluster ions, respectively. The peak size also decreased with the increase of the electron voltage and the electron current for ionization. This might be due to the formation of multiply charged cluster ions, which resulted in the dissociation of larger cluster ions by the Coulomb repulsion force.

In another case of producing liquid clusters at lower vapor pressures, He gas was used to mix up with vapors of liquid materials, and the mixed gases were injected into a high-vacuum region. Even if a vapor pressure of ethanol and water was less than $0.1 \mathrm{~atm}$, the liquid clusters were produced at the He gas pressures larger than $0.5 \mathrm{~atm}$. The peak size was 250 molecule per cluster for ethanol cluster ions and 1250 molecules per cluster for water cluster ions, respectively. The vapors of the liquid materials were cooled down by the collision with the He gas, which resulted in the effective formation of the liquid clusters even at lower vapor pressures.

${ }^{1}$ M. L. Cohen and W. Knight, Phys. Today, 42 (1990).

${ }^{2}$ W. D. Knight, W. A. de Heer, and W. A. Saunders, Z. Phys. D: At., Mol. Clusters 3, 109 (1986).

${ }^{3}$ M. D. Morse, Chem. Rev. (Washington, D.C.) 86, 1049 (1986).

${ }^{4} \mathrm{Ph}$. Buffat and J.-P. Borel, Phys. Rev. A 13, 2287 (1976).

${ }^{5}$ I. Yamada and G. H. Takaoka, Jpn. J. Appl. Phys., Part 1 32, 2121 (1993).

${ }^{6}$ T. Yamaguchi, J. Matsuo, M. Akizuki, C. E. Ascheron, G. H. Takaoka, and I. Yamada, Nucl. Instrum. Methods Phys. Res. B 99, 237 (1995).

${ }^{7}$ I. Yamada, Eur. Phys. J. D 9, 55 (1999).

${ }^{8}$ J. Momigny, H. Wankenne, and C. Krier, Int. J. Mass Spectrom. Ion Phys. 35, 151 (1980).

${ }^{9}$ M. N. Danchevskaya and S. N. Torbin, Int. J. Mass Spectrom. Ion Process. 56, 251 (1984).

${ }^{10}$ C. V. Robinson, E. W. Chung, B. B. Kragelund, J. Knudsen, R. T. Aplin, F. M. Poulsen, and C. M. Dobson, J. Am. Chem. Soc. 36, 8646 (1996).

${ }^{11}$ C.-C. Wu, J. C. Jiang, D. W. Boo, S. H. Lin, Y. T. Lee, and H.-C. Chang, J. Chem. Phys. 112, 176 (2000).

${ }^{12}$ G. H. Takaoka, H. Noguchi, T. Yamamoto, and T. Seki, Jpn. J. Appl. Phys., Part 2 42, L1032 (2003).

${ }^{13}$ G. H. Takaoka, H. Noguchi, K. Nakayama, Y. Hironaka, and M. Kawashita, Nucl. Instrum. Methods Phys. Res. B 237, 402 (2005).

${ }^{14}$ K. Sattler, J. Muhlbach, E. Rechnagel, and A. Reyes-Flotte, J. Phys. E 13, 673 (1980).

${ }^{15}$ O. F. Hagena and W. J. Obert, J. Chem. Phys. 56, 1793 (1972).

${ }^{16}$ O. F. Hagena, Rev. Sci. Instrum. 63, 2374 (1992).

${ }^{17}$ T. Seki, J. Matsuo, G. H. Takaoka, and I. Yamada, Nucl. Instrum. Methods Phys. Res. B 206, 902 (2003).

${ }^{18}$ T. Seki and J. Matsuo, Nucl. Instrum. Methods Phys. Res. B 216, 191 (2004).

${ }^{19}$ P. Scheier and T. D. Mark, J. Chem. Phys. 86, 3056 (1987).

${ }^{20}$ P. Scheier, A. Stamatovic, and T. D. Mark, J. Chem. Phys. 88, 4289 (1988).

${ }^{21}$ K. Sattler, J. Muhlbach, O. Echt, P. Pfan, and E. Rechnagel, Phys. Rev. Lett. 47, 160 (1981).

${ }^{22}$ J. G. Gay and B. J. Berne, Phys. Rev. Lett. 49, 194 (1982). 